\title{
Hölder continuity of solutions to a basic problem in the calculus of variations
}

\author{
Pierre Bousquet $^{\mathrm{a}}$, Carlo Mariconda $^{\mathrm{b}}$, Giulia Treu ${ }^{\mathrm{b}}$ \\ ${ }^{a}$ UMPA, ENS Lyon, 46 Allée d'Italie, 69007 Lyon, France \\ ${ }^{\mathrm{b}}$ Dipartimento di Matematica Pura e Applicata, Via Trieste 63, 35121 Padova, Italy \\ Received 20 June 2008; accepted 5 October 2008 \\ Available online 30 October 2008 \\ Presented by Haïm Brezis
}

\begin{abstract}
For the basic problem in the calculus of variations where the Lagrangian is convex and depends only on the gradient, we establish the continuity of the solutions when the Dirichlet boundary condition is defined by a continuous function $\phi$. When $\phi$ is Lipschitz continuous, then the solutions are Hölder continuous. To cite this article: P. Bousquet et al., C. R. Acad. Sci. Paris, Ser. I 346 (2008).
\end{abstract}

(C) 2008 Académie des sciences. Published by Elsevier Masson SAS. All rights reserved.

\section{Résumé}

Continuité hölderienne des solutions d'un problème de calcul des variations. Pour un problème de calcul des variations multidimensionnel, où le lagrangien convexe ne dépend que du gradient, on montre que la continuité de la fonction $\phi$ définissant la condition de Dirichlet au bord implique la continuité des minimiseurs sur l'adhérence du domaine. Lorsque $\phi$ est lipschitzienne, alors les minimiseurs sont hölderiens. Pour citer cet article : P. Bousquet et al., C. R. Acad. Sci. Paris, Ser. I 346 (2008).

(c) 2008 Académie des sciences. Published by Elsevier Masson SAS. All rights reserved.

\section{Version française abrégée}

On considère le problème $(\mathrm{P})$ de minimiser

$$
I: u \mapsto \int_{\Omega} F(\nabla u(x)) \mathrm{d} x
$$

sur $W_{0}^{1,1}(\Omega)+\phi$ où $\Omega$ est un ouvert borné de $\mathbb{R}^{n}, n \geqslant 2, F: \mathbb{R}^{n} \rightarrow \mathbb{R}$ est convexe et $\phi$ est continue sur $\mathbb{R}^{n}$. On suppose qu'il existe une solution $u$ de $(\mathrm{P})$ et on essaie de déterminer le lien entre la régularité de $\phi$ et celle de $u$ sur l'adhérence de $\Omega$, en l'absence de toute hypothèse de croissance sur le lagrangien. Notre travail s'inscrit dans la théorie des barrières, et fait suite à des travaux de Miranda, Cellina et Clarke sur la continuité lipschitzienne des

E-mail addresses: Pierre.Bousquet@umpa.ens-lyon.fr (P. Bousquet), Carlo.Mariconda@unipd.it (C. Mariconda), Treu@math.unipd.it (G. Treu). 
solutions. Cette-dernière exige des conditions dites de pente bornée, voire minorée. Si ces conditions ne sont pas satisfaites, est-ce que la seule continuité de $\phi$ implique la continuité des minimiseurs ? La réponse est donnée par le théorème suivant, valable pour des lagrangiens convexes mais pas nécessairement strictement convexes (dans ce cas, pour un lagrangien superlinéaire, on peut montrer [16] que l'ensemble des solutions a un plus grand et un plus petit élément au sens de (4)) :

Théorème 0.1. On suppose que $F$ est convexe et superlinéaire et que $\Omega$ est convexe. On note $w$ le plus grand ou le plus petit des minimiseurs de I sur $W_{0}^{1,1}(\Omega)+\phi$.

(i) Si $\phi$ est continue, alors $w$ est continue sur $\bar{\Omega}$.

(ii) Si on suppose de plus que $F$ est coercif d'ordre $p>1$ (i.e. $F(\xi) \geqslant a|\xi|^{p}+b$ pour certaines constantes a $>0$ et $b \in \mathbb{R})$ et que $\phi$ est lipschitzienne, alors $w$ est hölderienne sur $\bar{\Omega}$ d'ordre $\alpha:=\frac{p-1}{n+p-1}$.

Les conclusions (i) et (ii) restent vraies pour tout minimiseur de I si de plus les projections des faces de l'épigraphe de F sont uniformément bornées.

Ce théorème généralise à tous les ouverts convexes des résultats de Clarke (voir [5]). Les preuves détaillées des théorèmes apparaîtront dans [2] et [17].

\section{Introduction}

We consider the problem $(\mathrm{P})$ of minimizing

$$
I: u \mapsto \int_{\Omega} F(\nabla u(x)) \mathrm{d} x
$$

on $W_{0}^{1,1}(\Omega)+\phi$.

Here $\Omega$ is a bounded open subset of $\mathbb{R}^{n}, n \geqslant 2, F: \mathbb{R}^{n} \rightarrow \mathbb{R}$ is convex and $\phi$ is continuous on $\mathbb{R}^{n}$. We address the question of the regularity of any solution $u$ of $(\mathrm{P})$ on the closure $\bar{\Omega}$ of $\Omega$. More specifically, we present some recent results which emphasize the links between the regularity of $\phi_{\mid \Gamma}$ and the regularity of $u$. Our work is motivated by the following example due to Giaquinta [6]: let

$$
F\left(\xi_{1}, \ldots, \xi_{n}\right):=\sum_{i=1}^{n-1} \xi_{i}^{2}+\frac{1}{2} \xi_{n}^{4} .
$$

Then $F$ is a strictly convex function which satisfies the following growth hypotheses:

$$
C_{1}|\xi|^{p} \leqslant F(\xi) \leqslant C_{2}\left(1+|\xi|^{q}\right)
$$

with $p=2$ and $q=4$. However, a solution of the corresponding Euler equation (which is also the solution of (P) for the appropriate boundary condition $\phi$ ) is

$$
u\left(x_{1}, \ldots, x_{n}\right)=\frac{\sqrt{n-4}}{24} \frac{x_{n}^{2}}{\sum_{i=1}^{n-1} x_{i}^{2}} \quad(\text { for } n \text { sufficiently large }) .
$$

This function is singular along the line $x_{1}=\cdots=x_{n-1}=0$. Hence, the growth conditions (1) do not guarantee the continuity of a solution inside $\Omega$. In contrast, when $p=q$ in (1), the solution of (P) is continuous (see [8] and the references therein, and also [12] for certain extensions). Hence, the continuity of a minimizer cannot follow from growth assumptions on the Lagrangian except when these are rather restrictive.

Since the solution (2) is singular along a line, the boundary condition $\phi$ is singular at two points of the boundary $\Gamma$ of $\Omega$. Hence, the following question is natural: if $\phi$ were continuous (respectively Hölder continuous/Lipschitz continuous), were any solution of (P) continuous (respectively Hölder continuous/Lipschitz continuous)? Here, we do not assume any growth conditions on the convex function $F$. These questions are rooted in the theory of barriers where a now classical result (see $[19,3,14]$ ) asserts that if $\phi$ satisfies the bounded slope condition and $F$ is strictly 
convex, then any solution of (P) is Lipschitz continuous on $\bar{\Omega}$. We say that $\phi$ satisfies the bounded slope condition if $\phi_{\mid \Gamma}$ is the restriction to $\Gamma$ of a convex function defined on $\mathbb{R}^{n}$ and the restriction of a concave function defined on $\mathbb{R}^{n}$. Equivalently, there exists a constant $Q>0$ such that for any $\gamma \in \Gamma$, there exist $\zeta_{\gamma}^{ \pm} \in \mathbb{R}^{n},\left|\zeta_{\gamma}^{ \pm}\right| \leqslant Q$ which satisfy

$$
\phi(\gamma)+\left\langle\zeta_{\gamma}^{-}, \gamma^{\prime}-\gamma\right\rangle \leqslant \phi\left(\gamma^{\prime}\right) \leqslant \phi(\gamma)+\left\langle\zeta_{\gamma}^{+}, \gamma^{\prime}-\gamma\right\rangle \quad \forall \gamma^{\prime} \in \Gamma
$$

see $[9,10]$ for alternative definitions and characterizations of this condition.

On the one hand, many generalizations of this bounded slope condition have been considered recently, in connection with a better understanding of the comparison principles used in this context, see for instance [4,15] where the case of convex but not necessarily strictly convex Lagrangians are considered. On the other hand, Clarke (see [5]) has investigated the case when only half of the bounded slope condition is satisfied, namely when $\phi_{\mid \Gamma}$ is the restriction of a convex function defined on $\mathbb{R}^{n}$. This lower bounded slope condition plays a fundamental role in the proof of the following theorems. All these results are restricted to the case when $\Omega$ is convex, which we will assume from now on.

The Lagrangians $F$ we consider are not necessarily strictly convex. Hence, the problem (P) may have several solutions. To handle this situation, we introduce the two following assumptions on the Lagrangian:

(HF1) $F$ is superlinear; that is, $\lim _{|p| \rightarrow+\infty} \frac{F(p)}{|p|}=+\infty$.

(HF2) The projections on $\mathbb{R}^{n}$ of the faces of the epigraph of $F$ are uniformly bounded.

Assumption (HF2) means that for some constant $Q>0$, for any $\xi, \xi^{\prime} \in \mathbb{R}^{n}$, if there exists $\zeta$ in the convex subdifferential of $F$ at $\xi$ satisfying $F\left(\xi^{\prime}\right)=F(\xi)+\left\langle\zeta, \xi^{\prime}-\xi\right\rangle$, then $\left|\xi-\xi^{\prime}\right| \leqslant Q$.

Note also that (HF1) is satisfied when $F$ is coercive of order $p>1$ : there exist $a>0, b \in \mathbb{R}$ such that

$$
F(\xi) \geqslant a|\xi|^{p}+b .
$$

Assume that $F$ satisfies (HF1) or (HF2). If there exists at least one solution (this is automatically the case when $F$ is superlinear), then there exist two solutions of $(\mathrm{P}) w_{-}, w_{+} \in W^{1,1}(\Omega)$ (the maximum and minimum minimizers of $I$ ) such that

$$
w_{-}(x) \leqslant w(x) \leqslant w_{+}(x) \text { a.e. } x \in \Omega \text {, for any solution } w \text { of (P) (see [16]). }
$$

\section{Statement of the results}

Theorem 2.1. Assume that $F$ is convex and superlinear and that $\Omega$ is convex. Denote by $w$ the maximum or the minimum minimizer of $I$ on $W_{0}^{1,1}(\Omega)+\phi$.

(i) If $\phi$ is continuous, then $w$ is continuous on $\bar{\Omega}$.

(ii) If we assume further that $F$ is coercive of order $p>1$ and that $\phi$ is Lipschitz, then $w$ is Hölder continuous in $\bar{\Omega}$ of order $\alpha:=\frac{p-1}{n+p-1}$.

Conclusions (i) and (ii) above hold true for every minimizer of I if moreover the projections of the faces of the epigraph of $F$ are uniformly bounded.

Claim (i) extends [1] Theorem 5 which was only valid for uniformly convex sets; claim (ii) extends [5], Theorems 2.2 and 1.6 formulated in the case when $\Omega$ is a polyhedron and $\phi$ satisfies the lower bounded slope condition.

In the case when $F$ is not convex (but superlinear), conclusions (i) and (ii) above hold true for every minimizer of $I$ if the projections of the faces of the epigraph of the bipolar $F^{* *}$ of $F$ are uniformly bounded.

\section{Open problems}

Here, we assume that $F$ is convex and coercive and that $\Omega$ is convex. We denote by $w$ any solution of (P). 
1) Assume that $\phi$ is Hölder continuous of order $a<1$. Is it true that $w$ is Hölder continuous in $\Omega$ ?

2) Assume that $\phi$ is Lipschitz continuous. Is it true that $w$ is locally Lipschitz?

3) Consider the case of a non-convex (but regular) set $\Omega$.

In Claim 2, there is no hope for $w$ to be globally Lipschitz on $\bar{\Omega}$; see [5,1] where a counterexample is given when $F(\xi)=|\xi|^{2}$. On the contrary, Claim 1 holds true if $F(\xi)=|\xi|^{p}(p>1)$ even for general domains $\Omega$ (actually for those which satisfy the Wiener criterion; see e.g. [18, Corollary 4.20]). In the case when $p=2$ and for a convex set $\Omega$, it is shown in [20] that the solution, the harmonic function that coincides with $\phi$ on $\Gamma$, is Hölder continuous of the same order of $\phi$. Moreover, the solution is Hölder continuous if $F$ merely satisfies a polynomial growth condition and $\Omega$ is Lipschitz (see [7]). In the third open problem, the claims (i) and (ii) of Theorem 2.1 may be false if no regularity assumption is made on $\Omega$ (for instance, the case of a domain with a sharp inwardly directed spine is considered in [11]).

\section{Barriers and comparison principles}

In this section, we present several important tools in the proof of Theorem 2.1. We begin with the following comparison principle (see [16]):

Theorem 4.1. Assume that the convex map $F$ is superlinear or that the projections of the faces of the epigraph of $F$ are uniformly bounded. Let $\phi_{1}, \phi_{2} \in W^{1,1}(\Omega)$. When $F$ is not superlinear, we assume further that $(\mathrm{P})$ admits a solution on $W_{0}^{1,1}(\Omega)+\phi_{i}, i=1,2$. Let $w_{1}$ be the minimum minimizer of $I$ on $W_{0}^{1,1}(\Omega)+\phi_{1}$, and $w_{2}$ be any minimizer of $I$ on $W_{0}^{1,1}(\Omega)+\phi_{2}$. If $\phi_{1}, \phi_{2}$ satisfy

$$
\phi_{1} \leqslant \phi_{2} \quad \text { on } \Gamma \text { in the trace sense, }
$$

then $w_{1}(x) \leqslant w_{2}(x)$ a.e. $x \in \Omega$.

Theorem 4.1 has the following interesting consequence:

Corollary 4.2. Let $w$ be the maximum or the minimum minimizer of $I$ on $W_{0}^{1,1}(\Omega)+\phi$ where $\phi$ is assumed to be continuous on $\mathbb{R}^{n}$. Assume that there exist two continuous functions $l^{-}, l^{+}: \bar{\Omega} \rightarrow \mathbb{R}$ such that

$$
l^{-}(x) \leqslant w(x) \leqslant l^{+}(x) \quad \text { a.e. } x \in \Omega ; \quad l^{-}(x)=l^{+}(x)(=\phi(x)) \quad \text { for any } x \in \Gamma .
$$

Denote by $\omega:\left[0,+\infty\left[\rightarrow\left[0,+\infty\left[\right.\right.\right.\right.$ a modulus of continuity of $l^{-}$and $l^{+}$on $\bar{\Omega}$ :

$$
\left|l^{ \pm}(x)-l^{ \pm}(y)\right| \leqslant \omega(|x-y|) \quad \forall x, y \in \bar{\Omega} .
$$

Then $\omega$ is a modulus of continuity of $w$.

Proof. For any $h \in \mathbb{R}^{n}$, we have $\partial(\Omega \cap(\Omega-h)) \subset \partial \Omega \cup(\partial \Omega-h)$. Hence, we easily get that on $\partial(\Omega \cap(\Omega-h))$

$$
|\operatorname{tr} w(\cdot+h)-\operatorname{tr} w(\cdot)| \leqslant \max \left(\left|l^{+}(\cdot+h)-l^{+}(\cdot)\right|,\left|l^{-}(\cdot+h)-l^{-}(\cdot)\right|\right) \leqslant \omega(|h|) .
$$

Since $w$ and $w(\cdot+h) \pm \omega(|h|)$ are maximum (or minimum) minimizers on $\Omega \cap(\Omega-h)$, Theorem 4.1 implies that (6) remains true for almost every $x \in \Omega \cap(\Omega-h)$. This completes the proof of Corollary 4.2.

However, the main difficulty is the construction of two barriers $l^{ \pm}$. In Theorem $2.1, l^{ \pm}$are defined via the minimizers of an auxiliary variational problem (with the same Lagrangian, but different set $\Omega$ and boundary condition $\phi$, see $[2,17]$ for details).

Remark 4.1. The proof of Corollary 4.2 shows that if we replace the assumption (5) by the following one

$$
\text { there exists } \omega:[0,+\infty[\rightarrow[0,+\infty[\text { such that } \forall \gamma \in \Gamma, \quad|w(x)-\phi(\gamma)| \leqslant \omega(|x-\gamma|) \text { a.e. } x \in \Omega,
$$

then the same conclusion holds true: $\omega$ is a modulus of continuity of $w$ (see [13]). 
A third interesting tool is given by the following (see [2], proofs of Theorem 2 and Corollary 3):

Proposition 4.3. Let $w$ be a solution of $(\mathrm{P})$ on $W_{0}^{1,1}(\Omega)+\phi$. Assume that $\Omega$ is convex, that the convex map $F$ satisfies either (HF1) or (HF2) and that $\phi$ is continuous. If $w$ is continuous at $\Gamma$, then $w$ is continuous on $\bar{\Omega}$.

The continuity at $\Gamma$ means that for any $\gamma \in \Gamma, \lim _{x \in A, x \rightarrow \gamma} w(x)$ exists and is equal to $\phi(\gamma)$,

where $A$ denotes the set of Lebesgue points of $w$ in $\Omega$.

Proof. Let

$$
\omega(r):=\sup _{(x, \gamma) \in A \times \Gamma,|x-\gamma| \leqslant r}|w(x)-\phi(\gamma)| .
$$

Then (7) implies that $\lim _{r \rightarrow 0} \omega(r)=0$. By Remark 4.1, $\omega$ is a modulus of continuity of $w$. Hence, $w$ is uniformly continuous on $\bar{\Omega}$.

\section{References}

[1] P. Bousquet, On the lower bounded slope condition, J. Convex Anal. 14 (1) (2007) 119-136.

[2] P. Bousquet, Boundary continuity of solutions to a basic problem in the calculus of variations, submitted for publication.

[3] A. Cellina, On the bounded slope condition and the validity of the Euler Lagrange equation, SIAM J. Control Optim. 40 (2001/2002) 12701279.

[4] A. Cellina, Comparison results and estimates on the gradient without strict convexity, SIAM J. Control Optim. 46 (2007) $738-749$.

[5] F.H. Clarke, Continuity of solutions to a basic problem in the calculus of variations, Ann. Scuola Norm. Sup. Pisa Cl. Sci. 4 (2005) 511-530.

[6] M. Giaquinta, Growth conditions and regularity, a counterexample, Manuscripta Math. 59 (1987) 245-248.

[7] M. Giaquinta, E. Giusti, On the regularity of the minima of variational integrals, Acta Math. 148 (1982) 31-46.

[8] E. Giusti, Direct Methods in the Calculus of Variations, World Scientific Publishing Co., Inc., River Edge, NJ, 2003.

[9] P. Hartman, On the bounded slope condition, Pacific J. Math. 18 (3) (1966) 495-511.

[10] P. Hartman, Convex sets and the bounded slope condition, Pacific J. Math. 25 (1968) 511-522.

[11] I.N. Krol', V.G. Maz'ya, The absence of the continuity and Hölder continuity of the solutions of quasilinear elliptic equations near a nonregular boundary, Trans. Moscow Math. Soc. 26 (1974) 73-93.

[12] P. Marcellini, Regularity for some scalar variational problems under general growth conditions, J. Optim. Theory Appl. 90 (1) (1996) 161-181.

[13] C. Mariconda, G. Treu, Gradient maximum principle for minima, J. Optim. Theory Appl. 112 (2002) 167-186.

[14] C. Mariconda, G. Treu, Existence and Lipschitz regularity for minima, Proc. Amer. Math. Soc. 130 (2) (2002) 395-404.

[15] C. Mariconda, G. Treu, Lipschitz regularity for minima without strict convexity of the Lagrangian, J. Differential Equation 243 (2007) 388413.

[16] C. Mariconda, G. Treu, Local Lipschitz regularity of minima for a scalar problem of the calculus of variations, Commun. Contemp. Math., in press.

[17] C. Mariconda, G. Treu, Hölder regularity for a classical problem of the calculus of variations, submitted for publication.

[18] J. Malý, W. Ziemer, Fine Regularity of Solutions of Elliptic Partial Differential Equations, Mathematical Surveys and Monographs, vol. 51, Amer. Math. Soc., 1997.

[19] M. Miranda, Un teorema di esistenza e unicità per il problema dell' area minima in $n$ variabili, Ann. Scuola Norm. Sup. Pisa (3) 19 (1965) 233-249.

[20] S. Solimini, Simplified excision techniques for free discontinuity problems in several variables, J. Funct. Anal. 151 (1997) 1-34. 